\title{
Comparison of Accuracy for HKA by X-ray and Knee Motion Analysis System and the Relationships between HKA and Gait Posture
}

\author{
Hui Zhang \\ China Agricultural University \\ Yanan Chen \\ Shanghai Ocean University \\ Huiquan Jiang \\ Shanghai Ocean University \\ Wenqing Yan \\ Shanghai Sixth People's Hospital Lingang Campus, Shanghai Jiao Tong University \\ Yuanming Ouyang \\ Shanghai Sixth People's Hospital Lingang Campus, Shanghai Jiao Tong University \\ Wei Wang \\ Shanghai Sixth People's Hospital Lingang Campus, Shanghai Jiao Tong University \\ Yaru Liu \\ Shanghai Ocean University \\ Ying Zhou \\ Shanghai Sixth People's Hospital Lingang Campus, Shanghai Jiao Tong University \\ Shiyi Gu \\ Shanghai Sixth People's Hospital Lingang Campus, Shanghai Jiao Tong University \\ Hong Wan \\ Shanghai Sixth People's Hospital Lingang Campus, Shanghai Jiao Tong University \\ Axiang $\mathrm{He}$ \\ Shanghai Sixth People's Hospital Lingang Campus, Shanghai Jiao Tong University \\ Yanjie Mao \\ Shanghai Sixth People's Hospital Lingang Campus, Shanghai Jiao Tong University \\ Wanjun Liu ( $\square$ healthliuwanjun@126.com ) \\ Shanghai Sixth People's Hospital Lingang Campus, Shanghai Jiao Tong University
}

\section{Research Article}

Keywords: HKA , X-ray ,knee motion analysis system, gait posture

Posted Date: August 20th, 2021

DOI: https://doi.org/10.21203/rs.3.rs-798002/v1

License: (c) (i) This work is licensed under a Creative Commons Attribution 4.0 International License. Read Full License 


\section{Abstract}

This study is aimed at obtaining the accuracy of the lower limb mechanical axis (Hip-Knee-Ankle, HKA) by the six degrees of freedom (6DOF) of the knee and ground gait data and analyzing the correlation between the lower limb mechanical axis and 6DOF. We analyzed 3D knee kinematics during ground gait of 99 patients with KOA and 80 patients 6 months after the operations with the vivo infrared navigation 3D portable knee joint movement analysis system (Opti-Knee®, Innomotion Inc, Shanghai, China). Significant correlations with low coefficients ( $r$ $=-0.19, p=0.01$ ) between HKA value and anterior-posterior displacement for the whole cohort were found. There were significant correlations with moderate to high coefficients $(r=0.784$ to 0.976$)$ between the comparisons of HKA measured on the full-length alignment radiographs and 3D knee joint movement analysis system (Opti-Knee). The further linear correlation analysis showed that there was a significant correlation between the values of HKA measured by $X$-ray and movement analysis system $\left(R^{2}=0.90, p<0.01\right)$. Data with equivalent results as HKA could be provided by knee joint movement analysis system comparing with the conventional X-rays. Nonetheless, there was low significance between the HKA and ground gait data .

\section{Introduction}

Knee osteoarthritis (KOA) is a common joint disorder especially in the elderly and its incidence is rising ${ }^{1}$. There are various methods for evaluating KOA, mainly scoring systems ${ }^{2}$, imaging ${ }^{3,4}$ and reference standards ${ }^{5}$. Among them, radiographic film is the simplest, cheapest, and the main method for the diagnosis and evaluation of KOA. Lower limb alignment plays a significant role in the progress of KOA and is also an eminent indicator of load transmission. The most classic method of assessing the alignment of lower limbs is to measure the hip-knee-ankle angle (HKA), which is measured by full-length radiographs of the lower limbs covering the entire hip, knee, and ankle joints in the weightbearing position ${ }^{6}$. When arthritis developed, the arithmetic HKA algorithm can predict the constitutional alignment ${ }^{7}$. Phenotyping of HKA in young non-osteoarthritic knees provides better understanding of native alignment variability ${ }^{8}$. The HKA angle is associated with the subchondral trabecular bone microarchitecture which is related to KOA severity ${ }^{9}$. HKA demonstrates super diagnostic validity concerning medial and lateral joint space narrowing ${ }^{10}$. Standing HKA radiographs have been recognized as a key component of successful surgeries. Theoretically, the HKA angle is highly suitable as a preoperative planning parameter for High Tibial Osteotomy (HTO) to ensure optimal postoperative alignment ${ }^{11}$. It is used in a plane preoperative planning of HTO to predict osteotomy depth, open height and correction angle according to the magnitude of deformity ${ }^{12}$. HKA was compared between neutral mechanical alignment and residual varus after Total knee arthroplasty (TKA) to figure out the satisfactory survival rate ${ }^{13}$.

However, several studies have found an insignificant relationship between HKA and TKA survivorship in recent years ${ }^{13-16}$. Indeed, HKA cannot be the only indicator to evaluate the surgical effect, survival rate and patient satisfaction. HKA is not representative of the dynamic loading occurring during gait, unlike the gait analysis which is a promising technique for assessing the biomechanical changes of the patients' lower limbs objectively. There are lots of parameters to assess the gait in elder patients with KOA such as velocity, stride length, step width, swing/stance ratio, the smoothness of gait activity, and maximum angular velocity ${ }^{17}$. Gait analysis can also be used to evaluate early effectiveness by the post-operative gait of the patients after TKA including knee flexion range, stride length, cadence, and compensatory hip and ankle rotation range ${ }^{18}$. The classification model along with biomechanical features can be used as an extra tool for objective and repeatable KOA diagnosis, reflecting the key gait features of different grades of KOA and representing joint function from the early to the final stage of the disease ${ }^{19}$. To date, there have been no reports on the six degrees of freedom (6DOF) of the knee joint data to compare the effect of lower limb mechanical axis for patients with KOA.

The goal of the present study was two-fold: (1) to analyse HKA throughout the gait cycle by infrared-based navigation three-dimensional (3D) knee joint movement analysis system and obtain it's accuracy, (2) to figure out if the 6DOF of the knee joint data is related to lower limb mechanical axis. We hypothesized that the 3D knee joint movement analysis system could measure HKA, and that 6DOF of the knee joint data varied with HKA.

\section{Material And Methods}

\section{Subjects}

99 patients with KOA and 80 patients about six months after surgery (i.e. TKA, Unicompartmental Knee Arthroplasty (UKA), HTO, Distal Femoral Osteotomy (DFO) and Patellofemoral Arthroplasty (PFA)) from January 2019 to December 2019 in the Department of Joint Surgery at our hospital were selected. The exclusion criteria for patients included, (1) Patients without history of major trauma, surgery or knee-related symptoms. (2) Patients with rheumatoid arthritis, ankylosing spondylitis, and other autoimmune diseases. (3) Patients with knee joint tumor, infection, severe osteoporosis, and other diseases affecting osteotomy healing. (4) Patients with concurrent severe flexion contracture 
deformities. According to the lower limb anatomy (Hip-Knee-Ankle angle) by measuring on standing full-length radiographs, the pre-operative patients with $\operatorname{KOA}\left[\left(-6^{\circ}-6^{\circ}\right),\left(<-6^{\circ}\right.\right.$ and $\left.\left.>6^{\circ}\right)\right]$ and post-operative patients $\left[\left(-3^{\circ}-3^{\circ}\right),\left(<-3^{\circ}\right.\right.$ and $\left.\left.>3^{\circ}\right)\right]$ were divided into 2 groups respectively. This study was conducted based on the protocol approved by the Medical Ethics Committee of Shanghai Sixth People's Hospital, all methods were carried out in accordance with relevant guidelines and regulations, and informed consents were obtained from all participants. Patients participated in the experiment joined voluntarily and fully understood the clinical trial protocol.

\section{Admission check and surgical method}

After admission, routine preoperative examinations (CRP, ESR, liver and kidney function, electrocardiogram, etc.) and relevant examinations including bone density, CT and MRI scan of knee joints, full-length radiographs, short knee radiographs have been checked. According to the patients' symptoms, signs, bone density, X-ray, CT and MRI examinations, different patients received the corresponding surgical methods:

(1)TKA: knee osteoarthritis grade of Kellgren-Lawrence Grade III or above; Multiple compartment osteoarthritis; medial articular surface "bone to bone"; severe patellofemoral joint degeneration;

(2)UKA: Single-compartment osteoarthritis of the knee joint; without severe line of force; The anterior cruciate ligament (ACL) and collateral ligament are in good condition; Intact or mild degeneration of cartilage in the contralateral compartment and patellofemoral joint;

(3)HTO: The patients who are younger than 60 years and require large activities; Single-compartment osteoarthritis patients with poor knee joint force; No or mild osteoarthritis in the tibiofemoral and patellofemoral joints; No osteoporosis; Varus deformity originates from the tibial side;

(4)DFO: Varus deformity originates from the femoral side;

(5)PFA: Patients with simple and severe patellofemoral osteoarthritis; without coaxial bone distortion, poor alignment of the lower limbs or large varus and valgus angles;

All surgeries were carried out by highly experienced surgeons from the joint department.

\section{Gait analysis device}

3D knee joint movement analysis system, as the integrated system for dynamic and real-time examination of knee joint movement function, could provide doctors with three-dimensional 6DOF of the knee joint quickly and accurately. It could measure the stability of knee joints under different states of motion such as flat walking, squatting, uphill and rotation to evaluate the function of knee joints before and after the operation, and it will generate inspection reports immediately. These reports, combined with X-ray/ CT/ MRI and other imaging examination of joint structure, helped doctors evaluate the knee joint movement function of subjects objectively.

\section{Experimental procedure and functional assessment}

A vivo infrared-based navigation three-dimensional knee joint movement analysis system (Opti-Knee®, Innomotion Inc, Shanghai, China) was used to record and analyse the kinematic data of the knees in 6DOF of both knees one day before surgery and six months after surgery at random (Fig. 1A).

Before the test, the doors and windows were closed, curtains were drawn to block external light. All luminous objects in the room were removed to avoid the interference of external interference on the data. The subjects took off the pants and fully exposed both lower limbs. The subjects were told to raise their head and chest, look straight ahead, maintain a standard standing posture, with arms akimbo to prevent the markers from being blocked, and then performed the test system calibration. Two rigid plates, each with four infrared light-reflecting markers (OK_Marquer; Innomotion), were attached to the thighs and shanks with bandages. The 3D motion of the rigid plates was tracked by a stereo binocular infrared camera at a frequency of $60 \mathrm{~Hz}$ (Fig. 1B). A hand-held digitizing probe, with four infrared reflective light-reflecting markers, was used to identify femoral and tibial landmarks on the femur and tibia (Fig. 1C). Femoral and tibial landmarks included the trochanter major, condylus lateralis, condylus medialis, medial tibial plateau, lateral tibial plateau, medial malleolus, and lateral malleolus (Fig. 1D).

When the patients walked freely and normally, an integrated synchronous high-speed camera was collecting walking videos and gait data at a frequency of 60 frames per second for the 60 s to identify gait cycles and then calculate 3D knee kinematic parameters in real time. HKA measured by weight-bearing full-length $X$-ray images and 3D knee joint movement analysis were taken during the follow-up visit six months after surgery. 


\section{Statistical analysis}

All data processing and statistical analyses were undertaken with sigmaplot 14.0 (Systat Software Inc., San Jose, CA, USA) and SPSS 26.0 (IBM Corp., Armonk, NY, USA), respectively. 6DOF of different groups and HKA measured by 3D knee joint movement analysis system were computed and compared to the HKA values (X-ray) using the Student's t test and the linear correlation analysis. Pearson correlation coefficients between 6 DOF and HKA values were analysed for the whole cohort and the different groups separately. Significance was set at an alpha of 0.05 .

\section{Results}

\section{General information}

Demographic and anthropometric characteristics of all the groups are summarized in Table 1. Based on different HKA angles, preoperative patients were divided into group $A\left(-6^{\circ}-6^{\circ}\right)$ and group $B\left(<-6^{\circ}\right.$ and $\left.>6^{\circ}\right)$; patients six months after operation were divided into group $C\left(-3^{\circ}\right.$ $3^{\circ}$ )and group $\mathrm{D}\left(<-3^{\circ}\right.$ and $\left.>3^{\circ}\right)$. All demographic data are presented in Table 1.

\section{D knee kinematic alterations in ground gait}

Among the 177 knees analyzed in the present study, 99 (56\%) were preoperative and 80 (44\%) were post-operative. Comparisons of 3D knee kinematic parameters in the gait events between pre- and post-operation are shown in Table 2. The mean \pm standard deviation (SD) of 6DOF of the subjects during the ground gait (femur relative to tibia) is shown in it. According to different HKA angles, there are no statistical differences in the values of 6DOF ( $p>0.05$ ). Then the Pearson correlation coefficients between 6DOF and HKA values for the whole cohort and the various groups are showed.

There were significant correlations with moderate to high coefficients ( $r=0.784$ to 0.976$)$ between the comparisons of HKA measured on long leg alignment radiographs and optical motion capture system (Opti-Knee). In the further linear correlation analysis, $\mathrm{R}^{2}=0.90$ indicated that the model fitted well (Fig. 2).; the p value of the analysis of variance of the linear regression model was 0.000 , indicating the statistical significance between the independent variable "the values of HKA measured on the full-length alignment radiographs" and the dependent variable "the values of HKA measured on the 3D knee joint movement analysis system". The preoperative patients' data and postoperative values were $\left(R^{2}=0.94, p<0.01\right)$ and $\left(R^{2}=0.71, p<0.01\right)$ (Fig. 2).

The changes in the range of motion during gait are shown in Fig. 3. In both the stance phase and the swing phase, knee kinematic changes were similar in the groups ( $p>0.05$ ). Comparing the data of minor HKA angle groups, larger flexion/extension angle and varus/valgus angle and larger superior-inferior displacement were seen of the others, while the remaining 3DOF are opposite (Fig. 3 AB). The superior-inferior displacement of the post-operation was greater than the pre-operation during the stance phase, whereas the value of the post-operation was inferior to the pre-operation in the swing phase(Fig. $3 \mathrm{C}$ ).

HKA absolute variation was $3.36 \pm 5.72^{\circ}\left[-12.11^{\circ}-22.46^{\circ}\right]$ for the whole cohort. It was more for the pre-operation $\left(5.41 \pm 6.20^{\circ}\left[-12.11^{\circ}-\right.\right.$ $\left.\left.22.46^{\circ}\right]\right)$ and less for the post-operation $\left(0.83 \pm 3.76^{\circ}\left[-11.97^{\circ}-10.97^{\circ}\right], p=0.001\right)$. The difference were showed in $3 D$ knee kinematic parameters that the 6DOF (8.01 \pm 3.98 [0 - 19.7],13.55 \pm 5.65 [3.6 - 28.3], $2.08 \pm 1.04$ [0.6 - 5.5], $1.50 \pm 0.55$ [0.5 - 3] and 0.96 0.48 [0.3 - 2.5]) of minor HKA angle group less than that $(8.22 \pm 5.11$ [0 - 34.2], $13.94 \pm 6.29$ [2.6 - 30.8], $1.91 \pm 2.06$ [0.4 - 20.7], $1.72 \pm 2.09$ [0.4 -19.5$]$ and 1.00 \pm 0.52 [0.3 - 3.1]) of the other group respectively except flexion/extension angle (45.51 \pm 11.11 [10.8 - 62.6$]$ / $42.75 \pm 12.41$ [10.6 - 76.8]). The 6 DOF of the whole cohort and the various groups according to the HKA were not statistically significant.

Table 3 shows the Pearson correlation coefficients between 6DOF and HKA values for the whole cohort and the various groups. When comparing flexion/extension angle and HKA with Group A, significant differences were found $(r=0.29, p=0.042)$. There were significant correlations with low coefficients $(r=-0.19, p=0.01 ; r=-0.31, p=0.03)$ between HKA value and anterior-posterior displacement for the whole cohort and group C.

The Pearson correlation coefficients of HKA measured on long leg alignment radiographs and optical motion capture system (Opti-Knee) are shown in Table 4. There were significant correlations with moderate to high coefficients $(r=0.784$ to $0.976, p<0.001)$ between HKA measured by $\mathrm{X}$-ray and the 3D knee joint movement analysis system for the whole cohort, the pre-operation and the pre-operation groups.

Table 5 shows the Pearson correlation coefficients between 6DOF of the knee joint during the gait cycle. There was no significant correlation between varus/valgus angle and internal/external rotation, anterior-posterior displacement and superior-inferior displacement. And no 
significant differences between the internal/external rotation and superior-inferior displacement were found. In addition, there were significant correlations with low to moderate coefficients $(r=0.124$ to $0.494, p=0.01)$ in between the remaining 6DOF.

\section{Discussion}

No significant difference was observed between values of HKA measured on the full-length alignment radiographs and 3D knee joint movement analysis system (Opti-Knee). For reference, it was evidenced that the knee motion analysis could provide data with equivalent results as HKA.

The knee motion analysis uses the method of labeling in the body surface, recording the landmarks and calculating the distances between landmarks. By recording the femoral and tibial landmarks labeled by a hand-held digitizing probe with four infrared landmarks in each frame of the gait, the 3D positions were then measured as the distances between the landmarks in the femoral and tibial coordinate system. This method has certain consistency and credibility, which makes it different from other speculation methods. For example, standardized anteroposterior knee radiographs are insufficient to assess lower limb alignment of post-operative TKA ${ }^{20}$.

However, no significant difference was found between tested groups regarding the 6DOF based on data collected as Table 2. These outcomes are in agreement with the below findings in other currently available literature in related studies. It was found by Yan $\mathrm{W}$ that no significant statistical differences between the anterior cruciate ligament reconstruction knees and the corresponding contralateral normal knees were observed regarding the 5DOF ${ }^{21}$. Also, one other study had comparable results. According to Peixoto JG, there was no correlation between older women with bilateral knee and asymptomatic controls in relation to the step length and single support phase between lower limbs ${ }^{22}$. These three results collectively explained the insignificant difference in gait posture among different groups of mechanical axes after surgery. In addition to the above conclusion, there were significant correlations with low coefficients $(r=-0.19, p=0.01)$ only between anterior-posterior displacement and the HKA values for the whole cohort as shown in Table 3. This meant minor varus/ valgus alignment does not compromise gait posture; the 6DOF cannot be the indicator to evaluate the alignment of lower limbs.

In other related post-operation studies, the 6DOF in ground gait suggested that varied static HKA does not correspondingly predict kinematics

23. It has been shown that minor varus alignment does not compromise the mid- to long-term outcome of a medial UKA with no more than $7^{\circ}$ of varus by analyzing medial fixed-bearing UKAs ${ }^{24}$. This can be explained by that the post-operative mechanical axis has little effect on postoperative results according to IKS function scores or muscle strength et al. in previous studies ${ }^{15}$. Clément $\mathrm{J}$ noted that lower limb radiographic measures of coronal alignment have limited value for predicting dynamic measures during gait ${ }^{25}$. Similar results have been reported that during a systematic review and meta-analysis on PSI knee arthroplasty by A Mannan et al. ${ }^{16}$, Meta-analysis showed no obvious benefit for coronal alignment outcomes. Interestingly, comparing kinematic alignment TKA patients with mechanical alignment TKA patients, kinematic alignment in TKA reproduces normal gait better than mechanical alignment ${ }^{26}$.

It is, however, universal that patients after operations walk with an unnatural gait. Recent studies have shown that the single mechanical alignment often leads to significant anatomical modifications with a wide range of complex collateral ligament imbalances, which cannot be corrected by releasing collateral ligament ${ }^{13,26,27}$. A possible explanation for these unsatisfactory results may be related to the functional outcome of knee arthroplasty and osteotomy. There were significant differences in walking speed, cadence and stride length between UKA patients and healthy controls during level walking which means UKA cannot completely restore normal gait patterns during level walking clinically ${ }^{28}$. These outcomes were in agreement with the outcome of TKA. The knee kinematics during gait in TKA group improved. However, it could not fully reach the level of the healthy control group ${ }^{29}$. In comparison, neither type of knee arthroplasty restored knee kinematics to those of the non-operated side ${ }^{30}$. However, a more natural loading pattern can be achieved with UKA as compared to TKA ${ }^{12}$. Some gait features were also found to differ between post-HTO subjects and controls ${ }^{31}$. By using a dynamic metric of everyday activities, distinct gait differences between various arthroplasty types were established ${ }^{12}$.

Lower limb radiographic measures in the previous studies have limited value for predicting HKA, which revealed the irreplaceability of HKA. For patients, while measuring HKA by the 3D knee movement analysis system, the radiographic exposure was reduced. It reported HKA visually so that the clinician no longer needs to measure the angle in patients who had both full-length standing radiographs.

There were several limitations of this study. Firstly, there may be systematic errors by making comparisons for HKA of different individuals for both pre- and post- operation measurements. Secondly, we only compared gait posture and HKA in about six months after operations, with a lack of longitudinal follow-up. Thirdly, we only compared knee kinematic changes in patients without measuring healthy group as control.

\section{Conclusion}


In comparison with the conventional X-rays, infrared navigation based 3D portable knee joint movement analysis system could provide data with equivalent results for HKA and ground gait data simultaneously. However, regardless of whether ground gait data or HKA is known, either of them cannot be used to predict the value of the other.

\section{Declarations}

\section{Conflicts of interest statement}

The author(s) have no conflicts of interest relevant to this article.

\section{Funding statement}

The study was supported by the Shanghai Health and Hygiene Commission "Healthy aging" program (Grant No. 2020YJZX0120) and the Projects of Science and Technology Development Foundation of Pudong New District, Shanghai, China (Grant No. PKJ2018-Y54).

\section{Acknowledgements}

The authors thank the staff members of Shanghai Innomotion, Inc, for their kind assistance with our study. We would like to thank Chloe for her assistance with English language editing of the manuscript.

\section{Author contributions statement}

Conception and design of study: Wanjun Liu, Yuanming Ouyang, Yanjie Mao, Wei Wang; Acquisition of data: Hui Zhang, Yanan Chen, Huiquan Jiang, Wenqing Yan, Ying Zhou; Analysis and/or interpretation of data: Hui Zhang, Axiang He, Huiquan Jiang, Yaru Liu; Drafting the manuscript: Hui Zhang, Huiquan Jiang, Hong Wan, Shiyi Gu; Revising the manuscript critically for important intellectual content: Wanjun Liu, Yuanming Ouyang, Axiang He, Yanjie Mao.

\section{References}

1. Giwnewer, U., Rubin, G., Orbach, H. \& Rozen, N. [TREATMENT FOR OSTEOARTHRITIS OF THE KNEE]. Harefuah, 155, 403-406 (2016).

2. Bach, C. M. et al. Scoring systems in total knee arthroplasty. Clin Orthop Relat Res,184-196(2002).

3. Oo, W. M., Linklater, J. M. \& Hunter, D. J. Imaging in knee osteoarthritis. Curr Opin Rheumatol, 29, 86-95 (2017).

4. Oo, W. M. \& Bo, M. T. Role of Ultrasonography in Knee Osteoarthritis. J Clin Rheumatol, 22, 324-329 (2016).

5. Stephens, D. Egs General Assembly Conference. World Health Organization's international classification of functioning, disability and health - ICF.

6. Lu, Y. et al. Relationships between Morphological Changes of Lower Limbs and Gender During Medial Compartment Knee Osteoarthritis. Orthop Surg, 11, 835-844 (2019).

7. MacDessi, S. J., Griffiths-Jones, W., Harris, I. A., Bellemans, J. \& Chen, D. B. The arithmetic HKA (aHKA) predicts the constitutional alignment of the arthritic knee compared to the normal contralateral knee: a matched-pairs radiographic study. Bone Jt Open, 1, 339-345 (2020).

8. Hirschmann, M. T. et al. Phenotyping of hip-knee-ankle angle in young non-osteoarthritic knees provides better understanding of native alignment variability. Knee Surg Sports Traumatol Arthrosc, 27, 1378-1384 (2019).

9. Han, X. et al. Association between knee alignment, osteoarthritis disease severity, and subchondral trabecular bone microarchitecture in patients with knee osteoarthritis: a cross-sectional study. Arthritis Res Ther, 22, 203 (2020).

10. Moyer, R., Wirth, W. \& Eckstein, F. Sensitivity of different measures of frontal plane alignment to medial and lateral joint space narrowing: From the osteoarthritis initiative. Semin Arthritis Rheum, 45, 268-274 (2015).

11. Jiang, X. et al. HKA Angle-A Reliable Planning Parameter for High Tibial Osteotomy: A Theoretical Analysis Using Standing Whole-Leg Radiographs. J Knee Surg, https://doi.org/10.1055/s-0040-1712945 (2020).

12. Wiik, A. V. et al. The unicompartmental knee is the preferred side in individuals with both a unicompartmental and total knee arthroplasty. Knee Surg Sports Traumatol Arthrosc, 28, 3193-3199 (2020).

13. Zhang, Z. et al. Residual Mild Varus Alignment and Neutral Mechanical Alignment Have Similar Outcome after Total Knee Arthroplasty for Varus Osteoarthritis in Five-Year Follow-Up. J Knee Surg, 33, 200-205 (2020).

Page 6/11 
14. Abdel, M. P. et al. Effect of Postoperative Mechanical Axis Alignment on Survival and Functional Outcomes of Modern Total Knee Arthroplasties with Cement: A Concise Follow-up at 20 Years. J Bone Joint Surg Am, 100, 472-478 (2018).

15. Stucinskas, J. et al. Moderate varus/valgus malalignment after total knee arthroplasty has little effect on knee function or muscle strength. Acta Orthop, 86, 728-733 (2015).

16. Mannan, A., Smith, T. O., Sagar, C., London, N. J. \& Molitor, P. J. No demonstrable benefit for coronal alignment outcomes in PSI knee arthroplasty: A systematic review and meta-analysis. Orthop Traumatol Surg Res, 101, 461-468 (2015).

17. Mine, T. et al. Gait oscillation analysis during gait and stair-stepping in elder patients with knee osteoarthritis. J Orthop Surg Res, 14, 21 (2019).

18. Sun, M. et al. [Gait analysis after total knee arthroplasty assisted by three-dimensional printing navigation template]. Zhongguo Xiu Fu Chong Jian Wai Ke Za Zhi, 33, 953-959 (2019).

19. Kwon, S. B. et al. Identifying key gait features associated with the radiological grade of knee osteoarthritis., 27, 1755-1760 (2019).

20. Abu-Rajab, R. B. et al. Hip-Knee-Ankle Radiographs Are More Appropriate for Assessment of Post-Operative Mechanical Alignment of Total Knee Arthroplasties than Standard AP Knee Radiographs. J Arthroplasty, 30, 695-700 (2015).

21. Yan, W. et al. In vivo gait kinematics of the knee after anatomical and non-anatomical single-bundle anterior cruciate ligament reconstruction-a prospective study. Ann Transl Med, 7, 799 (2019).

22. Peixoto, J. G. et al. Analysis of symmetry between lower limbs during gait of older women with bilateral knee osteoarthritis. Aging Clin Exp Res, 31, 67-73 (2019).

23. Larose, G. et al. Can total knee arthroplasty restore the correlation between radiographic mechanical axis angle and dynamic coronal plane alignment during gait? Knee 26, 586-594(2019).

24. Vasso, M. et al. Minor varus alignment provides better results than neutral alignment in medial UKA. Knee, 22, 117-121 (2015).

25. Clément, J. et al. Hip-Knee-Ankle (HKA) angle modification during gait in healthy subjects. Gait Posture, 72, 62-68 (2019).

26. Blakeney, W. et al. Kinematic alignment in total knee arthroplasty better reproduces normal gait than mechanical alignment. Knee Surg Sports Traumatol Arthrosc, 27, 1410-1417 (2019).

27. Almaawi, A. M., Hutt, J. R. B., Masse, V., Lavigne, M. \& Vendittoli, P. A. The Impact of Mechanical and Restricted Kinematic Alignment on Knee Anatomy in Total Knee Arthroplasty. J Arthroplasty, 32, 2133-2140 (2017).

28. Kim, M. K., Yoon, J. R., Yang, S. H. \& Shin, Y. S. Unicompartmental knee arthroplasty fails to completely restore normal gait patterns during level walking. Knee Surg Sports Traumatol Arthrosc, 26, 3280-3289 (2018).

29. Bytyqi, D., Shabani, B., Cheze, L., Neyret, P. \& Lustig, S. Does a third condyle TKA restore normal gait kinematics in varus knees? In vivo knee kinematic analysis. Arch Orthop Trauma Surg, 137, 409-416 (2017).

30. Agarwal, A. et al. Comparison of gait kinematics in total and unicondylar knee replacement surgery. Ann R Coll Surg Engl, 101, 391-398 (2019).

31. Whatling, G. M. et al. High tibial osteotomy results in improved frontal plane knee moments, gait patterns and patient-reported outcomes. Knee Surg Sports Traumatol Arthrosc, 28, 2872-2882 (2020).

\section{Tables}

Table 1

Characteristics of the selected participants.

\begin{tabular}{|c|c|c|c|c|c|c|}
\hline & \multicolumn{2}{|l|}{ Pre } & \multirow[t]{2}{*}{$\mathrm{p}$} & \multicolumn{2}{|l|}{ Post } & \multirow[t]{2}{*}{$\mathrm{p}$} \\
\hline & Group A $\left(-6^{\circ}-6^{\circ}\right)$ & Group B $\left(<-6^{\circ}\right.$ and $\left.>6^{\circ}\right)$ & & Group $C\left(-3^{\circ}-3^{\circ}\right)$ & Group D $\left(<-3^{\circ}\right.$ and $\left.>3^{\circ}\right)$ & \\
\hline Sex(female/male) & $31 / 18$ & $38 / 12$ & 0.19 & $38 / 10$ & $27 / 5$ & 0.77 \\
\hline Age(y) & $65.98 \pm 7.45$ & $64.98 \pm 10.40$ & 0.58 & $63.94 \pm 7.19$ & $63.22 \pm 5.28$ & 0.63 \\
\hline Height(cm) & $160.10 \pm 6.60$ & $158.94 \pm 7.99$ & 0.43 & $159.81 \pm 8.27$ & $159.03 \pm 8.27$ & 0.68 \\
\hline Weight(kg) & $66.67 \pm 9.50$ & $67.80 \pm 10.38$ & 0.58 & $66.13 \pm 10.27$ & $65.31 \pm 11.27$ & 0.74 \\
\hline $\mathrm{BMI}\left(\mathrm{kg} / \mathrm{m}^{2}\right)$ & $26.04 \pm 3.67$ & $26.83 \pm 3.59$ & 0.28 & $25.92 \pm 3.72$ & $25.70 \pm 2.97$ & 0.78 \\
\hline
\end{tabular}

Table 2

Page $7 / 11$ 
Comparison of the whole cohort and the various groups HKA and 6DOF during a mean gait cycle.

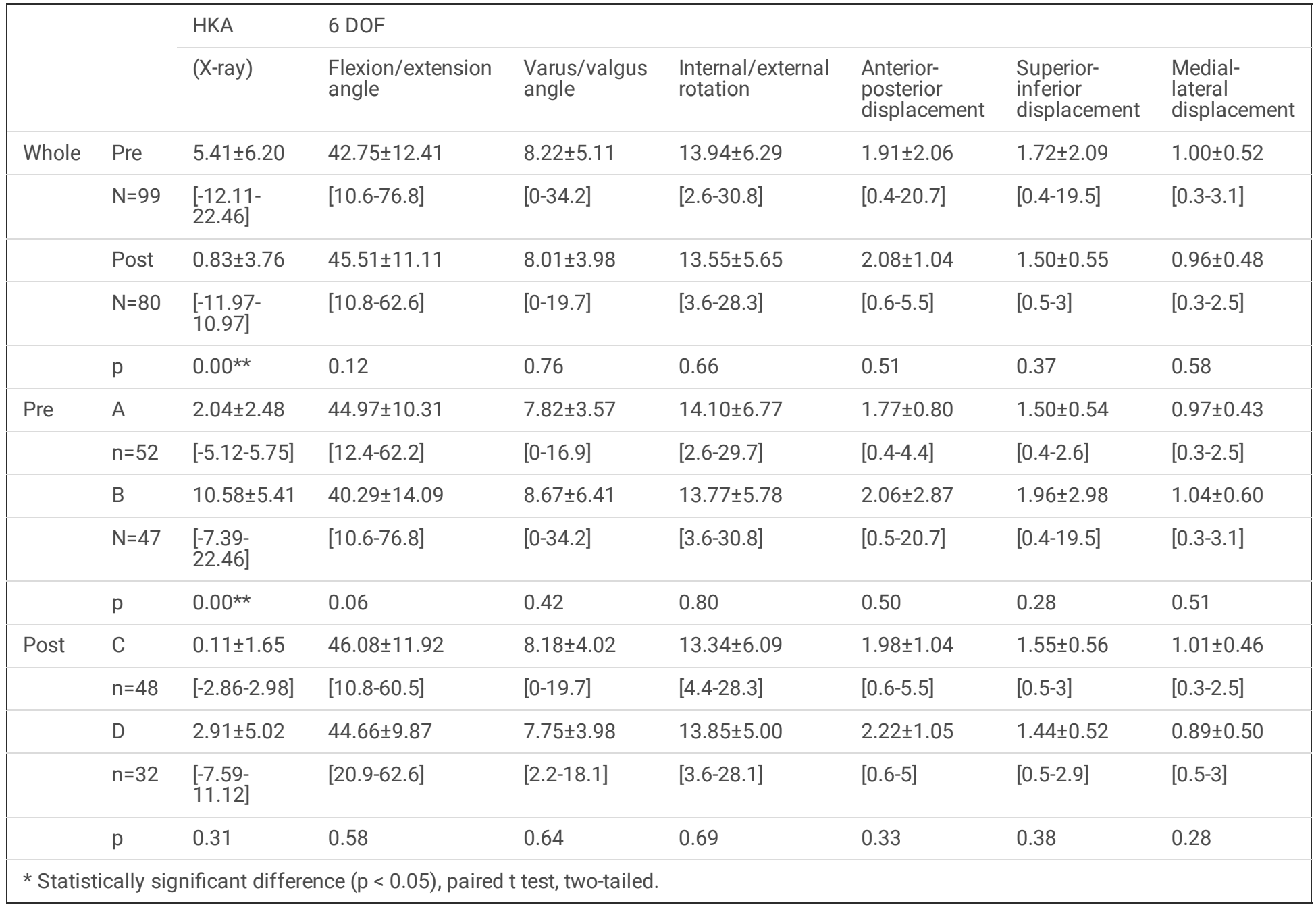

Table 3

Pearson correlation coefficients between HKA and 6DOF for the whole cohort and the various groups. 


\begin{tabular}{|c|c|c|c|c|c|c|c|}
\hline & HKA & $6 \mathrm{DOF}$ & & & & & \\
\hline & & $\begin{array}{l}\text { Flexion/extension } \\
\text { angle }\end{array}$ & $\begin{array}{l}\text { Varus/valgus } \\
\text { angle }\end{array}$ & $\begin{array}{l}\text { Internal/external } \\
\text { rotation }\end{array}$ & $\begin{array}{l}\text { Anterior- } \\
\text { posterior } \\
\text { displacement }\end{array}$ & $\begin{array}{l}\text { Superior- } \\
\text { inferior } \\
\text { displacement }\end{array}$ & $\begin{array}{l}\text { Medial- } \\
\text { lateral } \\
\text { displacement }\end{array}$ \\
\hline Whole & Pearson & -0.11 & 0.14 & 0.00 & $-0.19 *$ & -0.05 & -0.16 \\
\hline$N=179$ & Sig & 0.13 & 0.06 & 0.99 & 0.01 & 0.52 & 0.84 \\
\hline Pre & Pearson & -0.10 & 0.20 & -0.20 & -0.22 & -0.08 & 0.17 \\
\hline$N=99$ & Sig & 0.32 & 0.05 & 0.85 & 0.03 & 0.45 & 0.87 \\
\hline post & Pearson & -0.19 & 0.01 & -0.01 & -0.06 & -0.16 & -0.15 \\
\hline$N=80$ & Sig & 0.87 & 0.95 & 0.98 & 0.57 & 0.17 & 0.18 \\
\hline A & Pearson & $0.29 *$ & -0.74 & 0.12 & 0.10 & 0.24 & 0.05 \\
\hline$N=49$ & Sig & 0.042 & 0.61 & 0.41 & 0.50 & 0.09 & 0.71 \\
\hline B & Pearson & -0.09 & 0.23 & -0.06 & -0.31 * & -0.17 & -0.04 \\
\hline$N=50$ & Sig & 0.52 & 0.10 & 0.69 & 0.03 & 0.25 & 0.78 \\
\hline C & Pearson & 0.17 & 0.03 & -0.05 & -0.06 & -0.10 & 0.14 \\
\hline $\mathrm{N}=48$ & Sig & 0.24 & 0.84 & 0.72 & 0.69 & 0.52 & 0.34 \\
\hline D & Pearson & -0.10 & 0.02 & 0.00 & -0.19 & -0.20 & -0.26 \\
\hline$N=32$ & Sig & 0.58 & 0.91 & 0.99 & 0.31 & 0.28 & 0.15 \\
\hline
\end{tabular}

Table 4

Pearson correlation coefficients between HKA measured by X-ray and Opti-Knee.

\begin{tabular}{|c|c|c|c|c|c|c|c|c|}
\hline \multirow{3}{*}{ X-ray } & \multicolumn{3}{|l|}{ Pre } & \multicolumn{3}{|l|}{ Post } & \multirow[t]{2}{*}{ Whole } & \multirow{8}{*}{\begin{tabular}{|c} 
Table 5 \\
Pearson correlation \\
coefficients between \\
6DOF.
\end{tabular}} \\
\hline & Group A & Group B & & Group C & Group D & & & \\
\hline & $2.04 \pm 2.48$ & $10.58 \pm 5.41$ & $5.92 \pm 5.89$ & $0.11 \pm 1.65$ & $2.91 \pm 5.02$ & $1.27 \pm 3.71$ & $3.68 \pm 5.46$ & \\
\hline & {$[-5.12-5.75]$} & [-7.39-22.46] & {$[-7.39-22.46]$} & {$[-2.86-2.98]$} & {$[-7.59-11.12]$} & {$[-7.59-11.12]$} & [-7.11-22.46] & \\
\hline \multirow[t]{2}{*}{ Opti-knee } & $2.21 \pm 2.25$ & $10.68 \pm 5.61$ & $6.07 \pm 5.91$ & $0.17 \pm 1.75$ & $2.92 \pm 4.86$ & $1.31 \pm 3.65$ & $3.76 \pm 5.47$ & \\
\hline & {$[-4.60-7.10]$} & [-7.27-24.68] & {$[-7.28-24.68]$} & {$[-3.10-3.90]$} & {$[-7.45-11.05]$} & [-7.45-11.05] & [-7.45-24.68] & \\
\hline Pearson & $0.784^{\star \star}$ & $0.976^{\star \star}$ & $0.971^{\star \star}$ & $0.970^{\star *}$ & $0.789 \star \star$ & $0.84 * \star$ & $0.948^{\star \star}$ & \\
\hline Sig & 0.000 & 0.000 & 0.000 & 0.000 & 0.000 & 0.000 & 0.000 & \\
\hline
\end{tabular}




\begin{tabular}{|c|c|c|c|c|c|c|}
\hline & $\begin{array}{l}\text { Flexion/extension } \\
\text { angle }\end{array}$ & $\begin{array}{l}\text { Varus/valgus } \\
\text { angle }\end{array}$ & $\begin{array}{l}\text { Internal/external } \\
\text { rotation }\end{array}$ & $\begin{array}{l}\text { Anterior-posterior } \\
\text { displacement }\end{array}$ & $\begin{array}{l}\text { Superior-inferior } \\
\text { displacement }\end{array}$ & $\begin{array}{l}\text { Medial-lateral } \\
\text { displacement }\end{array}$ \\
\hline $\begin{array}{l}\text { Flexion/extension } \\
\text { angle }\end{array}$ & 1 & & & & & \\
\hline Varus/valgus angle & $0.212^{\star \star}$ & 1 & & & & \\
\hline $\begin{array}{l}\text { Internal/external } \\
\text { rotation }\end{array}$ & 0.494 * & 0.100 & 1 & & & \\
\hline $\begin{array}{l}\text { Anterior-posterior } \\
\text { displacement }\end{array}$ & $0.187^{\star}$ & -0.011 & 0.280 ** & 1 & & \\
\hline $\begin{array}{l}\text { Superior-inferior } \\
\text { displacement }\end{array}$ & $0.160^{\star}$ & 0.000 & 0.124 & $0.423^{\star \star}$ & 1 & \\
\hline $\begin{array}{l}\text { Medial-lateral } \\
\text { displacement }\end{array}$ & $0.321 * \star$ & $0.377^{\star \star}$ & $0.272^{\star \star}$ & $0.371^{\star \star}$ & $0.278^{\star \star}$ & 1 \\
\hline
\end{tabular}

\section{Figures}

\section{(A)}

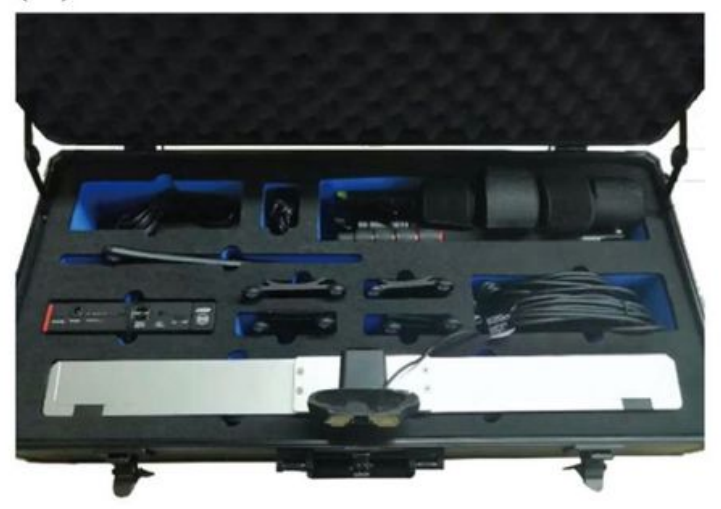

(B)

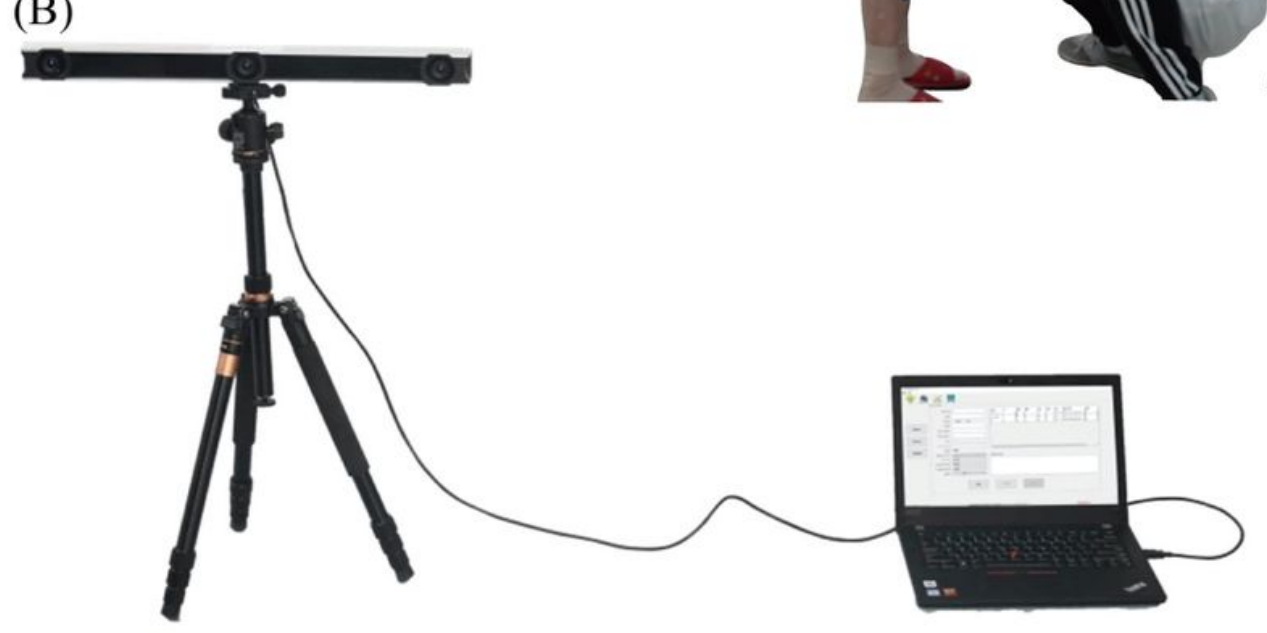

(C)

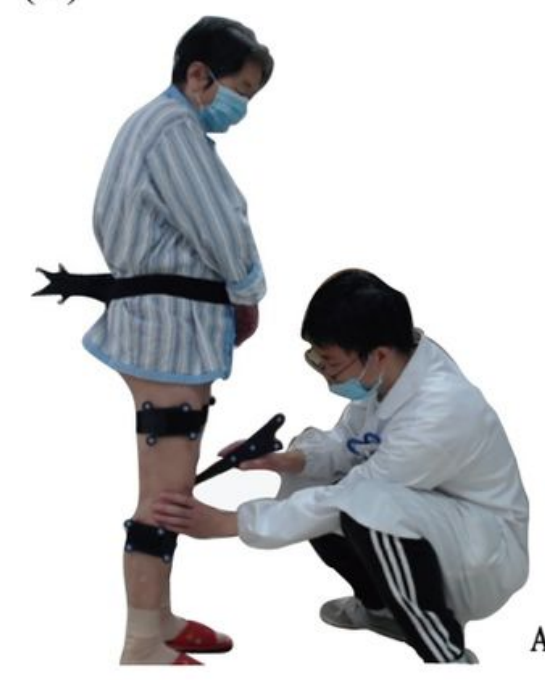

(D)

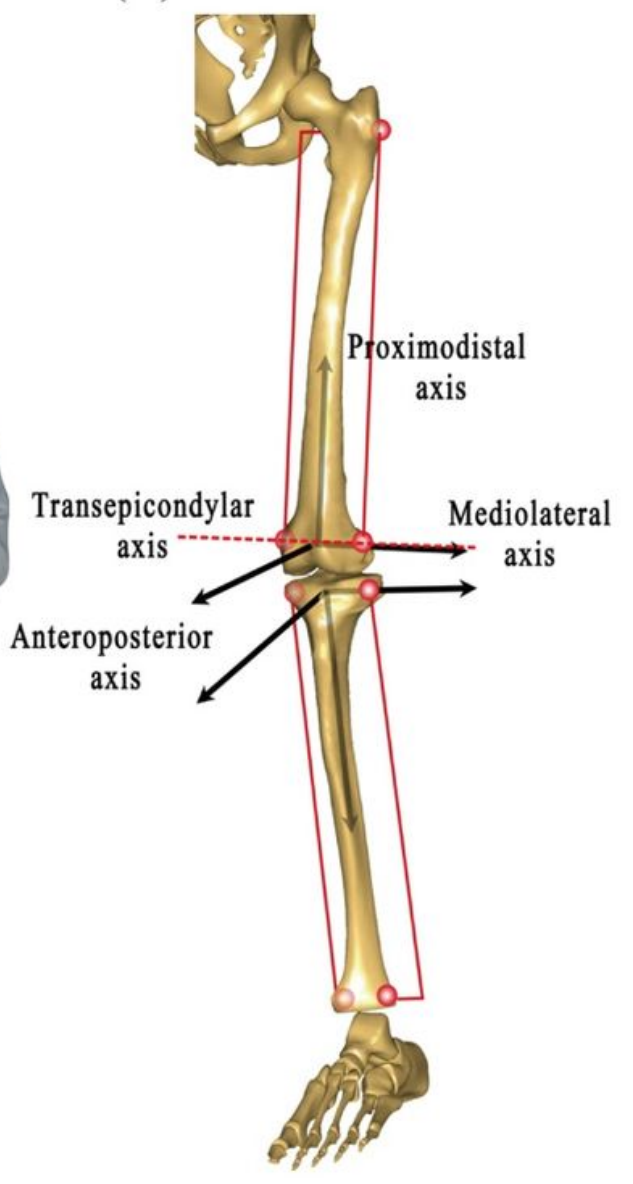

Figure 1

(A) Infrared navigation 3D portable knee joint movement analysis system. (B) Integrated dual stereo infrared camera and computer. (C) Identifying the femoral and tibial anatomical landmarks, the illustrated probe is pointing to the medial tibial plateau. (D) Definition of local femur and tibia coordinate systems. 

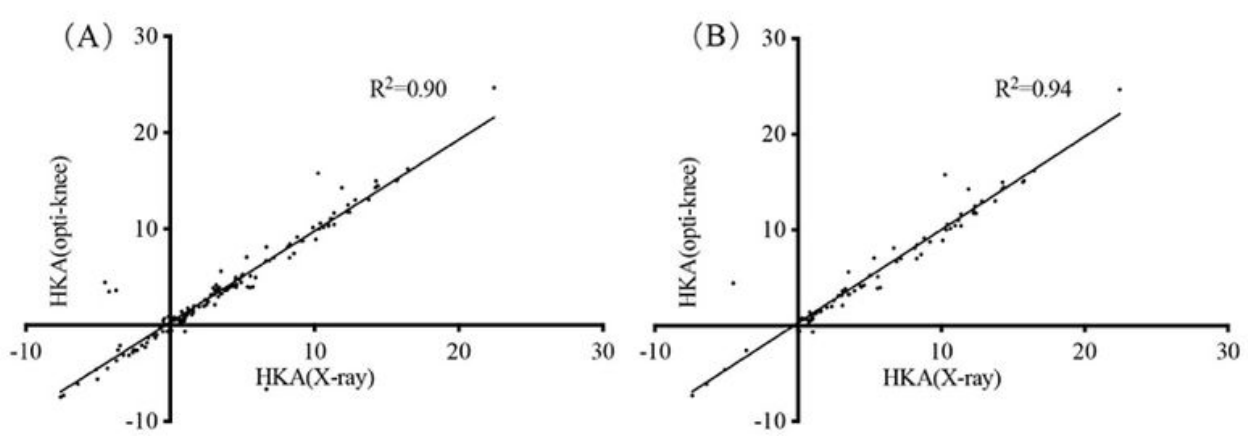

(C)

\section{Figure 2}

Linear correlation between the values of HKA measured on the full-length alignment radiographs (X-ray) and 3D knee joint movement analysis system (Opti-Knee), the whole (A), pre-operation (B) and post-operation (C).
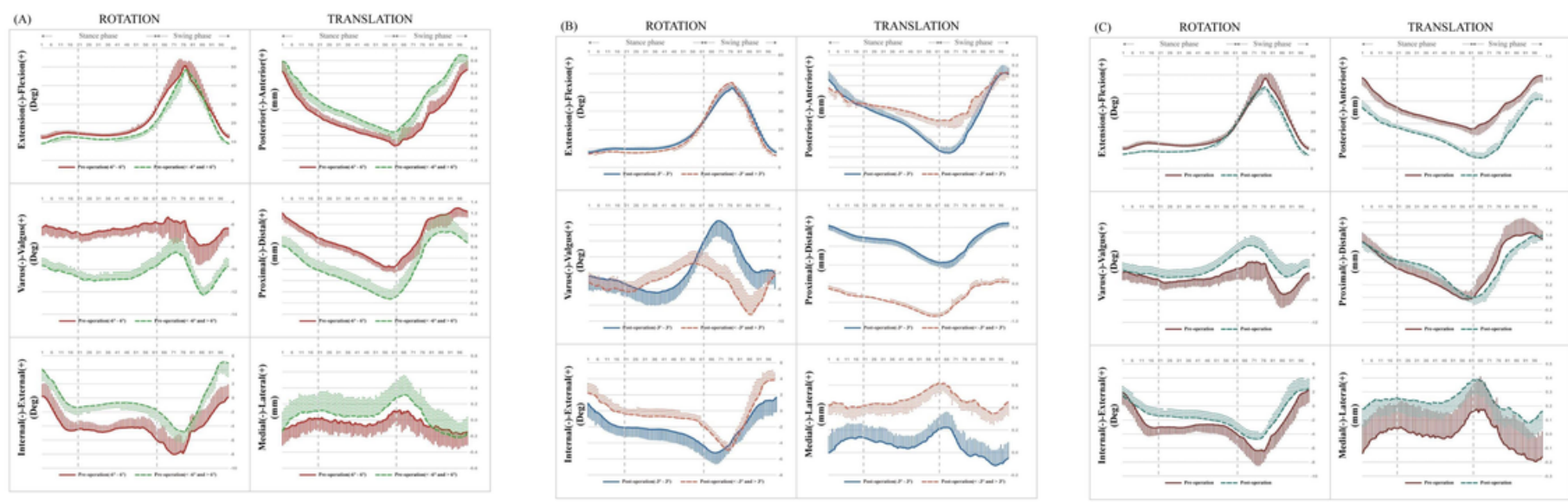

Figure 3

The 3D knee kinematic curves, including rotations and translations during ground walking gait, pre-operation (A), post-operation (B) and the whole (C). Ensemble of each walker were normalized from heel strike to next heel strike of the same foot as a gait cycle. The solid (dashed) curves and the lines above (below) them represent the mean and the SD (variability of these cycles) of gait cycle for two groups respectively. 